\title{
Inertial effects on acoustic Rayleigh streaming flow: Transient and established regimes
}

\author{
Virginie Daru ${ }^{\mathrm{a}, \mathrm{b}}$, Catherine Weisman ${ }^{\mathrm{b}, \mathrm{c}}$, Diana Baltean-Carlès ${ }^{\mathrm{b}, \mathrm{c}, *}$, Ida Reyt $^{\mathrm{a}, \mathrm{d}}$, \\ Hélène Bailliet ${ }^{\mathrm{d}}$ \\ a Arts et Métiers ParisTech, Lab. DynFluid, 151 Bd. de l'Hôpital, 75013 Paris, France \\ b LIMSI, CNRS, Université Paris-Saclay, Bât. 508, Rue John Von Neumann, Campus Universitaire, F-91405 Orsay Cedex, France \\ ' Sorbonne Universités, UPMC Univ Paris 06, UFR d'Ingénierie, 4 Place Jussieu, 75252 Paris Cedex 05, France \\ d Institut Pprime, CNRS - Université de Poitiers - ENSMA, ENSIP, 6 rue Marcel Doré, Bât. B17 - BP 633, 86022 Poitiers Cedex, France
}

\section{H I G H L I G H T S}

- Nonlinear inertial effects produce patterns different from DNS and experiments.

- Time evolutions of streaming cells near the wall and in the core are analyzed.

- The full streaming equations are solved numerically to obtain the established flow.

- Nonlinear inertial effects produce patterns different from DNS and experiments.

- Nonlinear inertial effects cannot explain the mutation of streaming at high levels.

\section{A B S T R A C T}

The effect of inertia on Rayleigh streaming generated inside a cylindrical resonator where a mono-frequency standing wave is imposed, is investigated numerically and experimen-tally. To this effect, time evolutions of streaming cells in the near wall region and in the resonator core are analyzed. An analogy with the lid-driven cavity in a cylindrical geometry is presented in order to analyze the physical meanings of the characteristic times.

Inertial effects on the established streaming flow pattern are then investigated numeri-cally using a code solving the time averaged Navier-Stokes compressible equations, where a mono-frequency acoustic flow field is used to compute the source terms.

It is shown that inertia of streaming cannot be considered as the leading phenomenon to explain the mutation of streaming at high acoustic levels.

Keywords:

Rayleigh streaming

Standing wave

Inertial effects

Nonlinear streaming

Transient evolution

\section{Introduction}

Acoustic streaming is often pointed out as being responsible for misunderstood thermal losses in thermoacoustic machines [1]. Among the different streaming flows found in such devices, Rayleigh streaming consists of a mean flow, of second order, which is produced by the interaction between the acoustic wave and a solid wall [2]. The pioneering work of Rayleigh was continued with the studies, among others, of Schlichting [3], Nyborg [4], Westervelt [5], Rudenko and Soluyan [6], yielding the steady streaming theory. Later, thermal effects were taken into account by Rott [7], the compressibility of the fluid by Qi [8] and more recently semi-analytical models adapted for thermoacoustic applications 

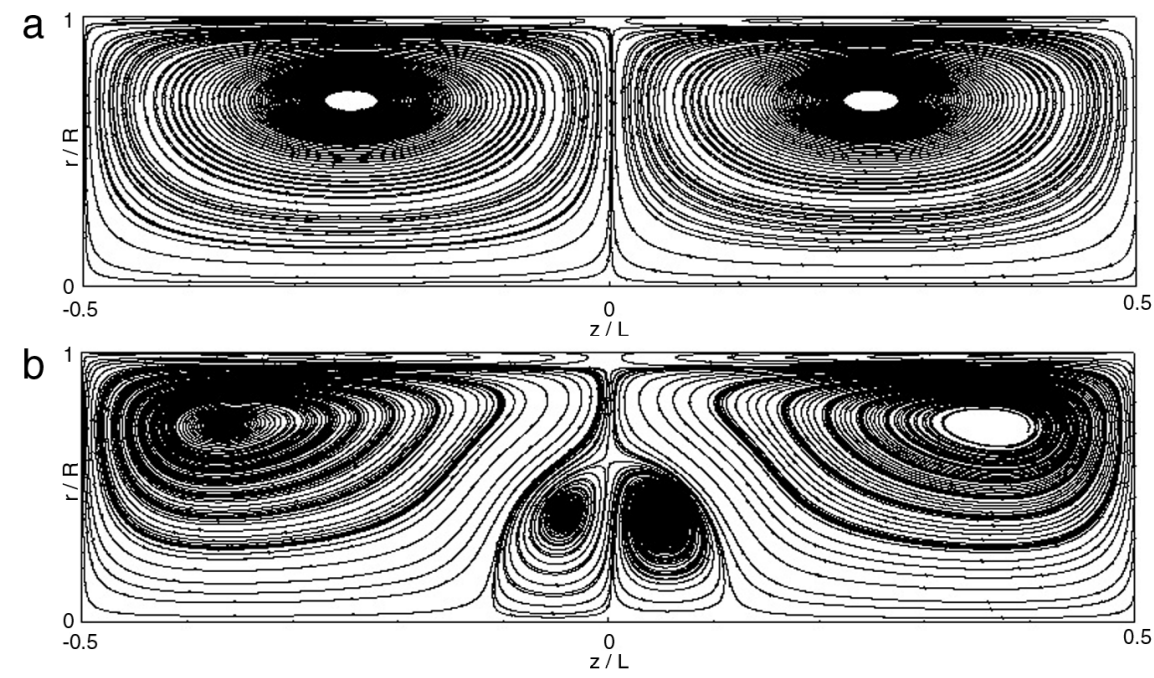

Fig. 1. Streamlines of streaming velocity fields without (a) and with (b) the extra contra-rotating cell (results of numerical simulations $N_{1}$ and $N_{2}$ ).

were developed (see for example $[9,10]$ ). These theoretical models, used to describe the streaming flow in the slow regime, are based on the method of successive approximations in powers of the Mach number, performed up to the second order and followed by time averaging over the acoustic period, yielding linear equations for streaming flow.

For high intensity acoustic waves, the streaming flow is referred to as nonlinear streaming or fast streaming. In this case, the effect of fluid inertia was first considered by [11-14] in unbounded or semi-infinite spaces, and more recently by Menguy and Gilbert [15] in resonators and thus for Rayleigh streaming. In the weakly nonlinear study proposed by [15], the method of successive approximations is performed up to the third order and separate solutions are obtained for the near-wall region (inner streaming) and in the resonator core (outer streaming). The global solution is obtained by applying matching conditions to the streaming velocity components. The inner streaming approximate equations were shown to remain linear in the weakly nonlinear case, while the outer streaming flow is affected by inertia [15].

Numerical simulations for acoustic Rayleigh streaming can be found in the literature starting in the 1990's. Two approaches are used: one is based on solving the full Navier-Stokes equations for low and high intensity waves and extract the mean flow from the instantaneous flow [16-18], and recently [19-22] and the other approach is based on solving streaming equations for the mean flow in the linear case [23,24] or in the nonlinear case [25]. Numerical simulations as well as experimental studies (for example [26,27]) in large resonators have shown the two regimes of streaming flows associated with standing waves: slow streaming similar to Rayleigh type streaming or fast streaming where the mean flow is strongly modified when the intensity of the acoustic wave is increased. The dimensionless number commonly used to differentiate between slow and fast streaming is the nonlinear Reynolds number $R e_{N L}$ [15], defined by $R e_{N L}=\left(M \times R / \delta_{v}\right)^{2}$, where $M$ is the acoustic Mach number, $M=U_{\max } / c_{0}$, with $U_{\max }$ the acoustic velocity amplitude at the velocity antinode and $c_{0}$ the speed of sound, $R$ the guide radius and $\delta_{v}=\sqrt{2 v / \omega}$ the acoustic boundary layer thickness, $\omega$ being the pulsation and $v$ the kinematic viscosity of the gas. Fig. 1(a) shows the streaming flow pattern (i.e. the streamlines for the steady mean velocity field, with the average performed over an acoustic period) in a half-plane containing the axis of a cylindrical waveguide of axial extension $\lambda / 2$ in the slow streaming regime (result from the numerical simulation $N_{1}$ further described in this paper). This pattern is symmetrical with respect to the guide center and is composed of four streaming cells: two inner toroidal cells close to the tube wall and two outer toroidal cells in the core. The established streaming flow behavior for high acoustic amplitudes (i.e. in the nonlinear regime of streaming) was previously analyzed numerically [19] (with a DNS code) and experimentally [28]: additional streaming cells were observed inside the resonator [29]. Fig. 1(b) shows the distorted streaming pattern (result from the numerical simulation $\mathrm{N}_{2}$ further described in this paper) in the fast streaming regime, when two new streaming cells are observed in the center of the guide, near the acoustic velocity antinode $(z / L=0)[19,28,29]$. Several sources could potentially be responsible for this mutation of streaming pattern: thermal effects were considered [26], as well as nonlinear propagation [29] and it was concluded that inertial effects on acoustic streaming could be the main source [29]. In the literature there is no study available that allows to conclude about the role of inertia in the behavior of streaming when the latter is "fast". The current study aims at investigating the hypothesis that inertia is responsible for the mutation of streaming flow at high levels by using both experimental and numerical results.

In Section 2, numerical methods and experimental setup are presented. Section 3 describes the establishment of slow and fast streaming using numerical and experimental observations. Time evolutions of inner and outer streaming cells are discussed. In order to analyze the associated physics, Section 4 shows the streaming flow equations and a scale analysis used to point out the leading parameters. The characteristic time scales are estimated according to the configuration parameters and compared with numerical and experimental results. 

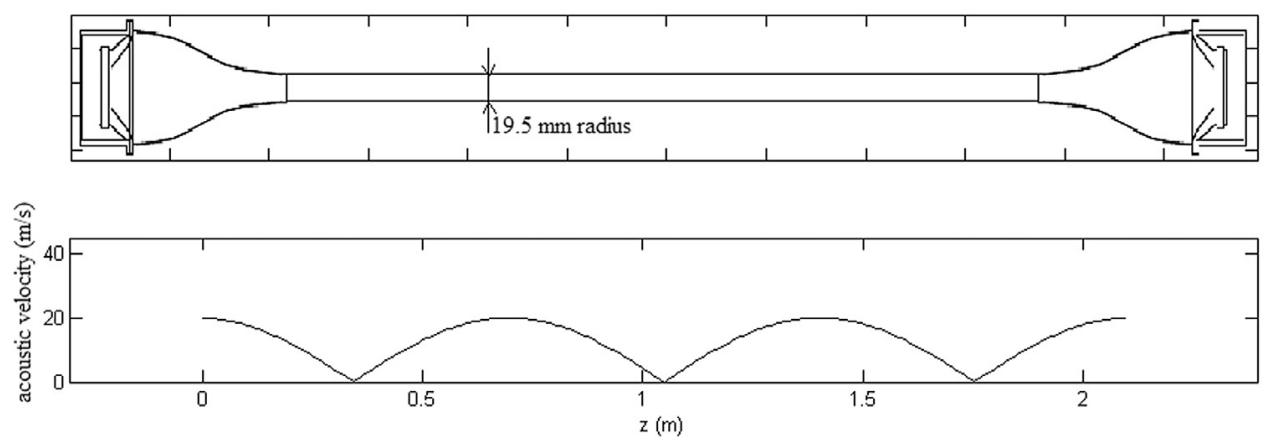

Fig. 2. Sketch of experimental setup and wave pattern for the working frequency.

In order to further isolate the driving effect of inner streaming on outer streaming, the problem of a flow inside a cylindrical tube with prescribed axial velocity on the walls is solved and analyzed in Section 5. Then the role of inertia on the established nonlinear streaming flow patterns is examined (Section 6) by solving the time-averaged isentropic NavierStokes compressible equations (AMS), where a mono-frequency acoustic flow field is used to compute the different terms that are sources of streaming flow. The effect of increasing the amplitude of the acoustic flow field on the streaming flow is analyzed. The results are compared with the predictions of the asymptotic model developed in [15] for the weakly nonlinear regime.

\section{Methods and set-up}

In this section, the experimental setup and the numerical configurations and methods used to investigate the effect of inertia during the establishment of the streaming flow and on the steady state flow are presented briefly (see [29] for more detail). The experimental and numerical configurations are very different in physical dimensions and in frequency but it was checked in [29] that they are asymptotically similar for acoustic and streaming flow. It was shown indeed that the same flow behavior is to be expected if $M \ll 1, R e_{a c} \gg 1$ and $S h \ll 1$ (where $R e_{a c}=\frac{1}{2}\left(\frac{\lambda}{\pi \delta_{v}}\right)^{2}, S h=\frac{\delta_{v}}{R}, \lambda$ being the acoustic wavelength). Both experimental and numerical configurations match these conditions (see Table 1 in [29]).

\subsection{Experimental set-up}

The set-up used to study acoustic streaming consists of a cylindrical glass tube of radius $R_{\exp }=19.5 \mathrm{~mm}$ filled with air at atmospheric pressure and at ambient temperature (Fig. 2). In order to reach high acoustic levels, the acoustic sound wave is generated by two loudspeakers (one at each end of the guide), tuned at the same frequency, that are driven in opposite phase. Those loudspeakers are connected to the main part of the guide via connecting tubes designed to avoid separation effects related to the singularities in change of section. The total length of the wave guide is $L_{\text {exp }}=2.13 \mathrm{~m}$ and the system is tuned at a frequency $f_{\exp }=240 \mathrm{~Hz}$ so that to excite the third mode of the system. This choice of working frequency allows to perform measurements on a streaming cell away from singularities. The set-up geometry prevents the wave distortion even at high levels.

A wave generator provides the loudspeakers input signal, whose frequency and amplitude are controlled, as well as a trigger reference signal, used to synchronize the laser Doppler measurement system. Velocity measurements are started when the acoustic field is switched on in order to measure the transient evolution of the streaming velocity field. Those measurements are performed at different locations within the streaming cells. The laser system used and the processing of the laser signal are described in [28].

\subsection{Numerical methods}

For the numerical calculation, we consider a cylindrical tube of length $L$ and radius $R$, initially filled with air, considered as an ideal gas. An acoustic standing wave is initiated by shaking the tube in the axial direction $(z)$ with given amplitude and frequency, so that a harmonic velocity law is imposed, $\mathbf{V}(t)=V(t) \vec{e}_{z}$, with $V(t)=z_{p} \omega \cos (\omega t)$, $\omega$ being the angular frequency and $z_{p}$ the amplitude of the tube displacement.

The flow verifies the compressible Navier-Stokes equations expressed in the moving frame attached to the tube, with an additional forcing source term depending on $\mathbf{V}$ :

$$
\left\{\begin{array}{l}
\frac{\partial \rho}{\partial t}+\nabla \cdot(\rho \mathbf{v}) \quad=0 \\
\frac{\partial \rho \mathbf{v}}{\partial t}+\nabla \cdot(\rho \mathbf{v} \otimes \mathbf{v})+\nabla p=\nabla \cdot(\overline{\bar{\tau}})-\rho \frac{d \mathbf{V}}{d t}
\end{array}\right.
$$


Table 1

Parameters of different presented cases ( $N$ : numerical, $E$ : experimental).

\begin{tabular}{lrrlll}
\hline Name & $f(\mathrm{~Hz})$ & $R / \delta_{v}$ & $R / L$ & $U_{\max }(\mathrm{m} / \mathrm{s})(z=0)$ & $R$ \\
\hline$N_{1}$ & 20000 & 50 & 0.09 & 0.5 & 0.005 \\
$N_{2}$ & 20000 & 50 & 0.09 & 61.83 & 0.18 \\
$E_{2}$ & 240 & 138 & 0.027 & 23 & 80.84 \\
\hline
\end{tabular}

where $p$ is the pressure, $\rho$ is the density, $\mathbf{v}=u \vec{e}_{z}+v \vec{e}_{r}$ is the flow velocity, $\overline{\bar{\tau}}=-\frac{2}{3} \mu(\nabla \cdot \mathbf{v}) \overline{\bar{I}}+2 \mu \overline{\bar{D}}$ the viscous stress tensor of a Newtonian fluid, $\overline{\bar{D}}$ the strain tensor, $\mu$ the dynamic viscosity which is supposed to be constant. In this formulation we have neglected the bulk viscosity since $\frac{M^{2}}{S h^{2} R e_{a c}^{2}} \ll 1[30]$.

We consider an isentropic state law for the ideal gas, given by

$$
p-p_{0}=c_{0}^{2}\left(\rho-\rho_{0}\right)
$$

where $p_{0}, \rho_{0}$ and $c_{0}$ are respectively the constant initial pressure, density and sound velocity. Therefore the energy equation is decoupled from the other equations and we only solve the mass and momentum equations (Eqs. (1) and (2)), excluding thermal effects in our study.

No slip conditions are used on the solid boundary $r=R$, i.e. $\mathbf{v}(r=R, z)=0$. On the axis $r=0$, symmetry conditions are used, i.e. $\mathbf{v}(r=0, z)=u(z) \vec{e}_{z}$ with $\frac{\partial u}{\partial r}(r=0, z)=0$. On the vertical boundaries $z=0, L$, symmetry conditions are also used, i.e. $\mathbf{v}(r, z=0, L)=v(r) \vec{e}_{r}$ with $\frac{\partial v}{\partial z}(r, z=0, L)=0$.

Due to the geometry, shock waves are generated in the simulations but it was checked that they are of small intensity and do not affect drastically the streaming flow [19,29]. The presence of harmonics will also be addressed in Section 6 of the present article.

The unsteady equations (Eq. (1)) are solved numerically using third order finite difference schemes [19,29]. The mean (streaming) flow is obtained from calculating a simple mean value for each physical quantity (velocity, pressure, density) over an acoustic period.

For simulations, the reference air pressure is $p_{0}=101325 \mathrm{~Pa}$ and the density is $\rho_{0}=1.2 \mathrm{~kg} / \mathrm{m}^{3}$. The reference speed of sound is $c_{0}=343.82 \mathrm{~m} / \mathrm{s}$. The air viscosity is $\mu=1.795 \times 10^{-5} \mathrm{~kg} \mathrm{~m}^{-1} \mathrm{~s}^{-1}$. Resonant conditions are imposed, for which $L=\lambda / 2$. Numerical values are $f_{\text {num }}=20000 \mathrm{~Hz}$, so that $\lambda / 2=8.396 \mathrm{~mm}$ and the acoustic period is $T_{\text {num }}=50 \times 10^{-6} \mathrm{~s}$. All results presented below are obtained using a regular mesh of rectangular cells composed of 500 points in the axial direction, and of $5 \times R / \delta_{v}$ points in the radial direction. The time step $\delta t$ is fixed equal to $8 \times 10^{-9} \mathrm{~s}$.

\section{Establishment of acoustic streaming}

In order to study the establishment of the streaming flow generated by a sinusoidal standing wave independently from the transient of its acoustic source, the numerical code described in Section 2.2 is used in the following modified manner: for time stepping during the transient of acoustics, at the end of each acoustic period, the instantaneous fields are reinitialized by subtracting the mean flow over that period. Hence, the streaming flow is calculated here only once the acoustics is established, in order to dissociate the transient of streaming from the establishment of acoustics. Of course this cannot be done in experiments so we chose to discuss only experimental results for which the establishment of streaming has its own time scale, which is not the case of experimental results at low levels.

The different case parameters are summarized in Table 1 . Subscript 1 is used to denote slow streaming and subscript 2 is used to denote fast streaming.

\subsection{Slow streaming}

Figs. 3 and 4 address the case of slow streaming flow (for $N_{1}$ ). The established streaming flow pattern is very regular and corresponds to the representation in Fig. 1(a). Fig. 3 shows the time evolution of the streaming pattern: contours of axial streaming velocity are displayed (with color code) and streamlines are superimposed on the right half plane section. On Fig. 3(b) are also displayed two specific locations that will be used for further investigations: $I\left(r=R-3 \delta_{v}, z=L / 4\right)$ representing the place of maximum inner streaming velocity and $O_{c}(r=0, z=-L / 4)$ representing the center of the plane section and the place of maximum outer streaming velocity in the slow regime. Note that the aspect ratio (height/length) of the displayed figures is equal to 0.25 which is much larger than the actual aspect ratio (height/length $=0.09$ ) so that both inner and outer streaming are visible. One can observe that the establishment of the streaming flow occurs in several stages: The inner streaming cell is established first (Fig. 3(a)) and then does not evolve very much. The magnitude of the outer streaming velocity increases on a longer time scale (Fig. 3(b), (c) and (d)). This is in agreement with the results of previous numerical simulations [25].

The time evolution of the streaming velocity at some specific points can also be displayed. Fig. 4(a) shows the time evolution of the axial streaming velocity at I (see Fig. 3(b)). Fig. 4(b) shows the time evolution of the axial streaming velocity 


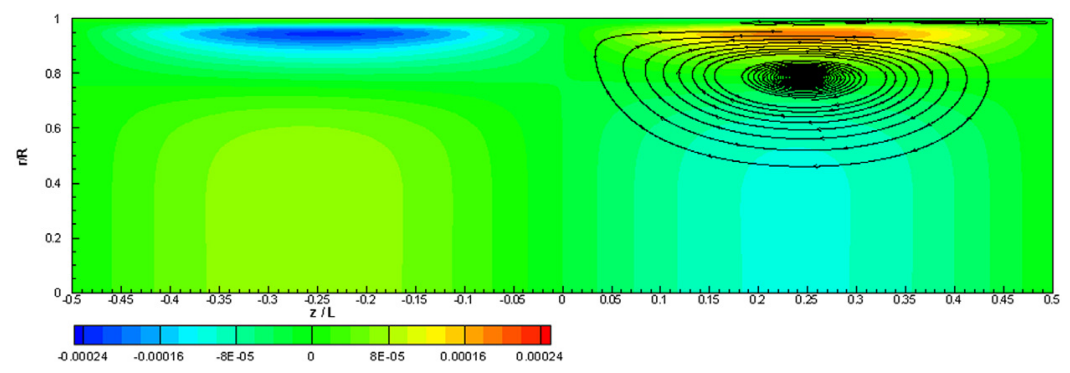

(a) $t=5.75 \times 10^{-3} \mathrm{~s}$.

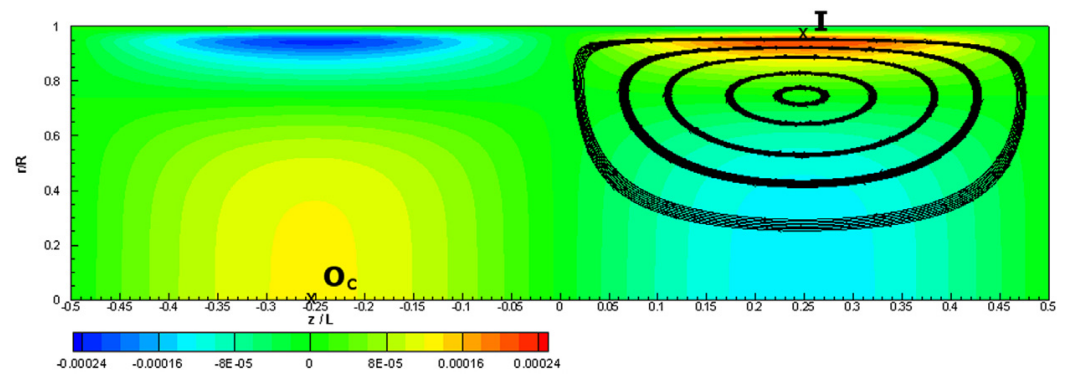

(b) $t=6.25 \times 10^{-3} \mathrm{~s}$.

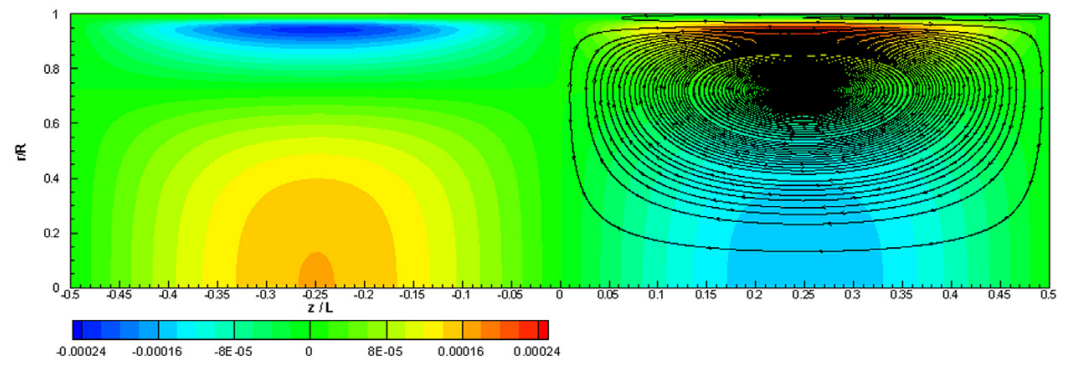

(c) $t=6.75 \times 10^{-3} \mathrm{~s}$.

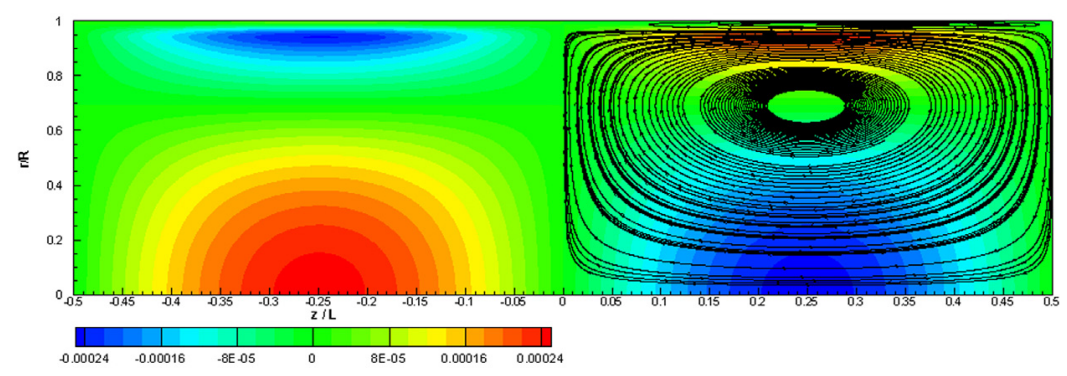

(d) $t=1 \times 10^{-2} \mathrm{~s}$.

Fig. 3. Contours of the axial streaming velocity (with color codes in $\mathrm{m} / \mathrm{s}$ ) at different times during the transient evolution of $N_{1}$. Streamlines are superimposed on the right half plane section (real aspect ratio height/length $=0.09$ ). (For interpretation of the references to color in this figure legend, the reader is referred to the web version of this article.)

at the center of the plane section $O_{c}$ (see Fig. 3(b)). In agreement with Fig. 3 the inner streaming (Fig. 4(a)) is established much faster than outer streaming (Fig. 4(b)). As expected from Rayleigh calculations [2], the steady state values at $O_{I}$ and $O_{c}$ are roughly equal.

In the experiments, the establishment of acoustics at low amplitudes is very long (see Fig. 13 of [28]). In this case, the evolution of streaming is intrinsically linked to that of acoustics and the associated establishment time scales, determined by fitting an $\exp (-t / \tau)$ law to experimental data, are very close $\left(\tau_{a c}=190 \mathrm{~s}\right.$ and $\left.\tau_{s t}=233 \mathrm{~s}\right)$. As will be shown in the next section, acoustics' transient is much shorter for high amplitudes ( $\tau_{a c}$ is of the order of tenths of seconds) so that the establishment of streaming can be observed for different locations in the wave guide. 

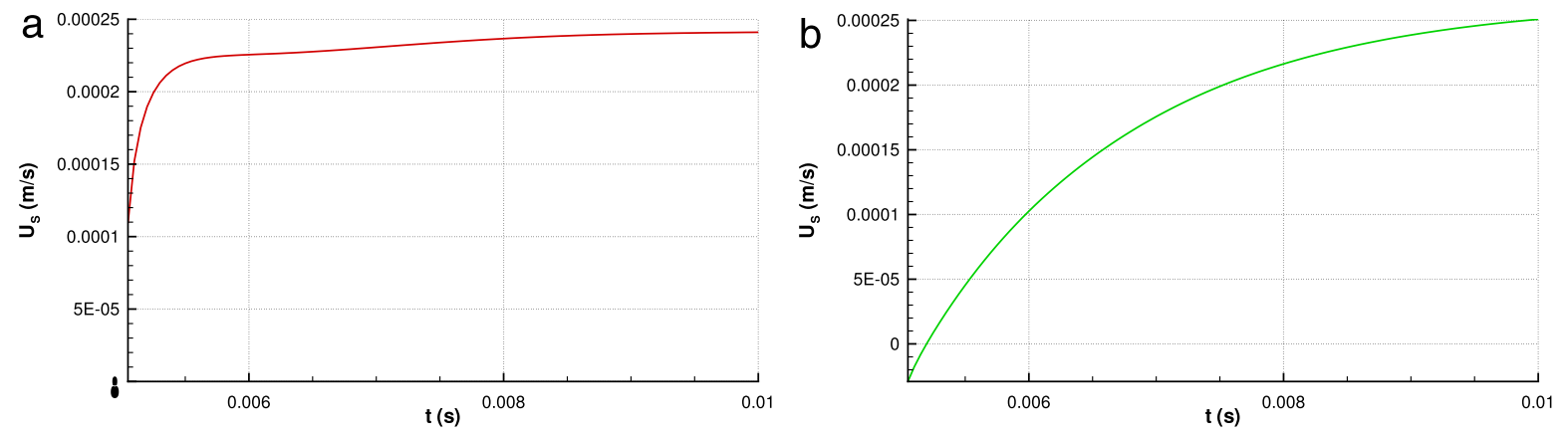

Fig. 4. Time evolution $N_{1}$. Axial streaming velocity at (a) $I$ and (b) $O_{c}$.

\subsection{Fast streaming}

Figs. 5-7 address cases of fast streaming flow (for $N_{2}$ and $E_{2}$ respectively). The established streaming flow pattern is strongly distorted with respect to the slow streaming case, and two extra contra-rotating streaming cells are present near the acoustic velocity antinode (see Fig. 5(d)), in agreement with Fig. 1(b).

Fig. 5 shows the time evolution of the streaming patterns: contours of axial velocity are displayed on the left, and streamlines are displayed on the right. On Fig. 5(d) are also displayed two specific locations that will be used for further investigations: $O_{e}$ representing the place on the axis corresponding to the maximum velocity inside the extra contra-rotating cell and $O_{c \text {-exp }}$ representing the location on the axis, close to the position $z=L / 4$, where measurements were performed to characterize the transient of the main outer streaming cell (both locations are on the right half side of the resonator). Here also the aspect ratio of the displayed figures is increased so that both inner and outer streaming are visible. One can observe that the establishment of the streaming flow occurs in several stages:

The inner streaming cell is established first, typically after $3 \times 10^{-3} \mathrm{~s}$ (see Fig. 5(a)) and then does not evolve very much. It is not as symmetrical as in the case of slow streaming (Fig. 3(a)), the cell centers being shifted towards the resonator closed ends (acoustic velocity node).

The magnitude of the outer streaming velocity increases on a longer time scale, and the main outer cell centers progressively shift towards the resonator closed ends (Fig. 5(b), (c) and (d)). Conversely to the slow streaming case, the maximum velocity of the outer streaming cell is smaller than that of inner streaming, as can be seen on the color code.

The emergence of the extra contra-rotating cell happens last (Fig. 5(d)).

On Fig. 6 are presented numerical time evolutions of the streaming velocity at $I, O_{c}$ (defined in the previous section, Fig. 3(b)) and at the place on the axis corresponding to the maximum velocity inside the extra contra-rotating cells $O_{e}$ (refer to Fig. 5(d)). First, this figure show that the time evolution of the inner streaming velocity at $I$ (Fig. 6(a)) is similar to the case of slow streaming (similar time scale).

Conversely in the core, the velocity increase for the location $O_{c}$ (Fig. 6(b)) depicts clearly an inflection point so that two characteristic time scales can be distinguished. Finally for the location $O_{e}$ (Fig. 6(c)) the velocity is first positive before becoming negative meaning that the contra-rotating cell appears after a certain time. A third time scale emerges from this time evolution. Note that the appearance of the extra cell does not modify the time evolution of streaming outside the extra cell (see Fig. 6(a) and (b)).

On Fig. 7 are presented experimental time evolutions for inner streaming at location I (Fig. 7(a)), for outer streaming on the axis $r=0$ at location $O_{c \text {-exp }}$ (Fig. 7(b)) and at the extra contra-rotating cell center $O_{e}$ (Fig. 7(c)) for the fast streaming case E2. Similar behavior to numerical results for equivalent positions can be observed on the experimental measurements and several distinct time scales are visible. The inner streaming is established first. Time evolution of outer streaming at $O_{c \text {-exp }}$ shows a distinct time scale. The magnitude of the contra-rotating cell maximum velocity is very small but the establishment of the extra streaming cell at $O_{e}$ is on a distinguished time scale from the last two.

In the experiment $E_{2}$ the established value in the inner cell $\left(U_{s} \simeq 0.5 \mathrm{~m} / \mathrm{s}\right.$ at $\left.I\right)$ is much greater than the established value in the core of the guide $\left(U_{s} \simeq 0.01 \mathrm{~m} / \mathrm{s}\right.$ at $O_{c \text {-exp }}$ ), as can be seen on Fig. 7. Similar observations were made on the simulation results in Fig. 5(b). Therefore, both experiments and numerical simulations show that nonlinear phenomena have very small influence on the streaming velocity in the boundary layer.

In summary, for slow streaming flow, only one time scale is necessary to describe the dynamics of streaming in the core of the waveguide. On the contrary for fast streaming flow, three different time scales were observed numerically and two experimentally in the core of the waveguide. At this stage the results seem to indicate that inner streaming flow is hardly modified by nonlinear effects (inertia included) at high levels. On the contrary the outer streaming is very strongly modified. This is in agreement with Menguy and Gilbert findings [15] which stated that inertia affects outer streaming but not inner streaming. However, it is necessary first to clearly identify the effect of inertia on the establishment of streaming. At this stage one cannot indeed assess whether inertial effects are truly responsible for the emergence of the new streaming cell. 


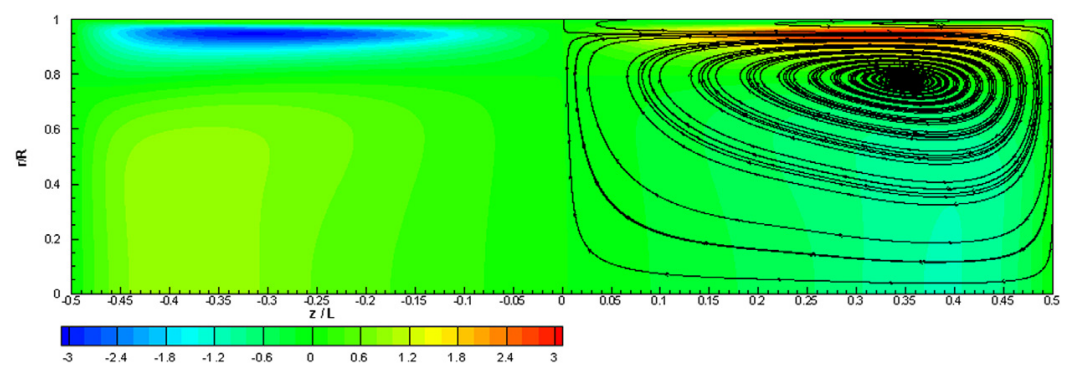

(a) $t=2.75 \times 10^{-3} \mathrm{~s}$.

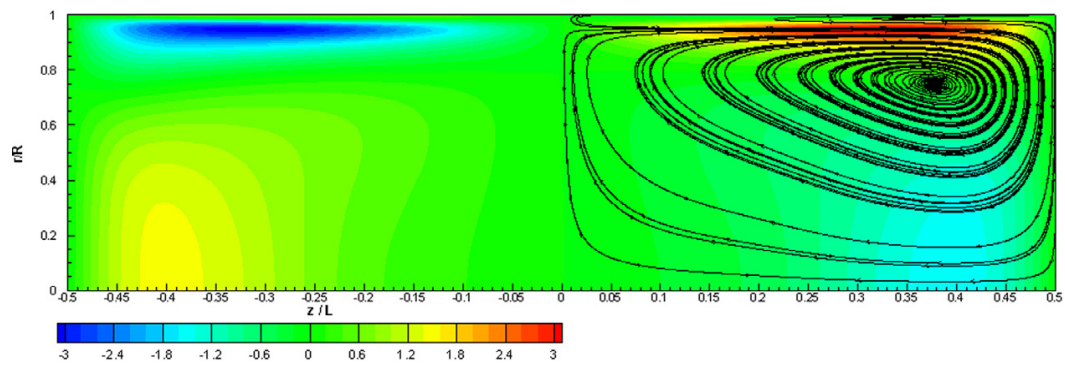

(b) $t=3.25 \times 10^{-3} \mathrm{~s}$.

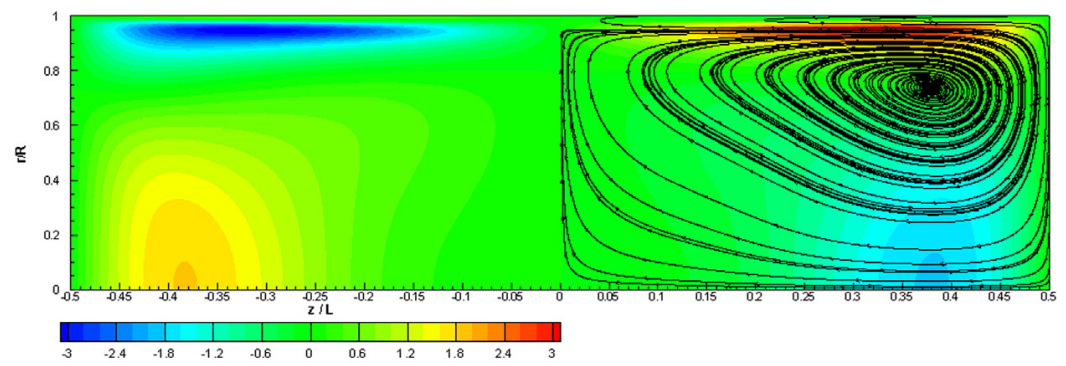

(c) $t=3.75 \times 10^{-3} \mathrm{~s}$.

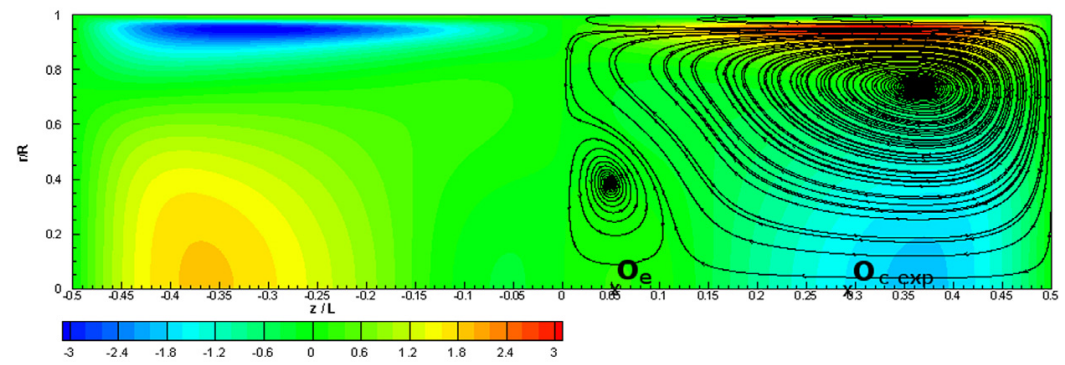

(d) $t=1 \times 10^{-2} \mathrm{~s}$.

Fig. 5. Contours of the axial streaming velocity (with color code) at different times during the transient evolution of $N_{2}$. Streamlines are superimposed on the right half plane section (real aspect ratio height/length $=0.09$ ). (For interpretation of the references to color in this figure legend, the reader is referred to the web version of this article.)

In the next section, the equations governing the streaming flow are presented along with a scale analysis in order to exhibit governing dimensionless numbers and time scales for the establishment of inner and outer streaming flows. The expected time scales for the configuration under study are then calculated and compared with fits to the curves shown in the present section.

\section{Scale analysis of slow and fast streaming flow}

In order to analyze the dynamics of the streaming flow and particularly the competition between diffusion at the origin of streaming and convection associated to nonlinear inertial effects, a scale analysis based on the full equations governing 

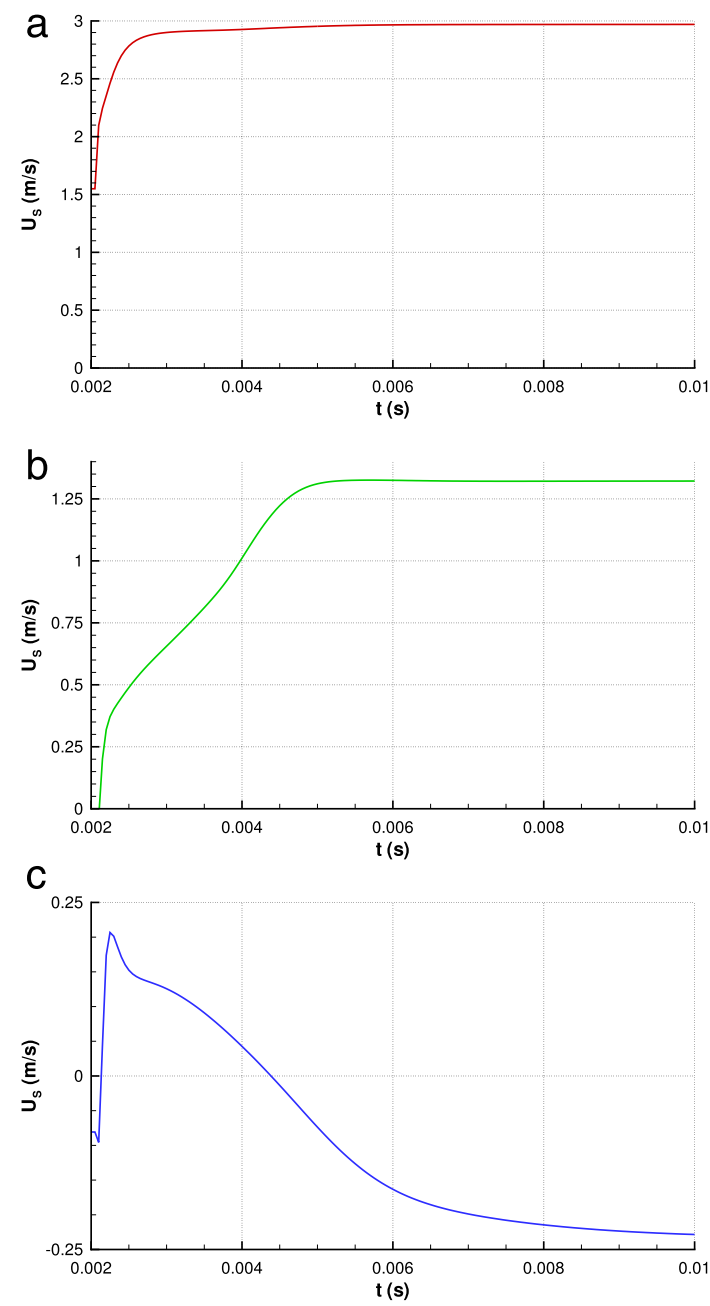

Fig. 6. Time evolution $N_{2}$. Axial streaming velocity at (a) $I$ (b) $O_{c}$ and (c) $O_{e}$.

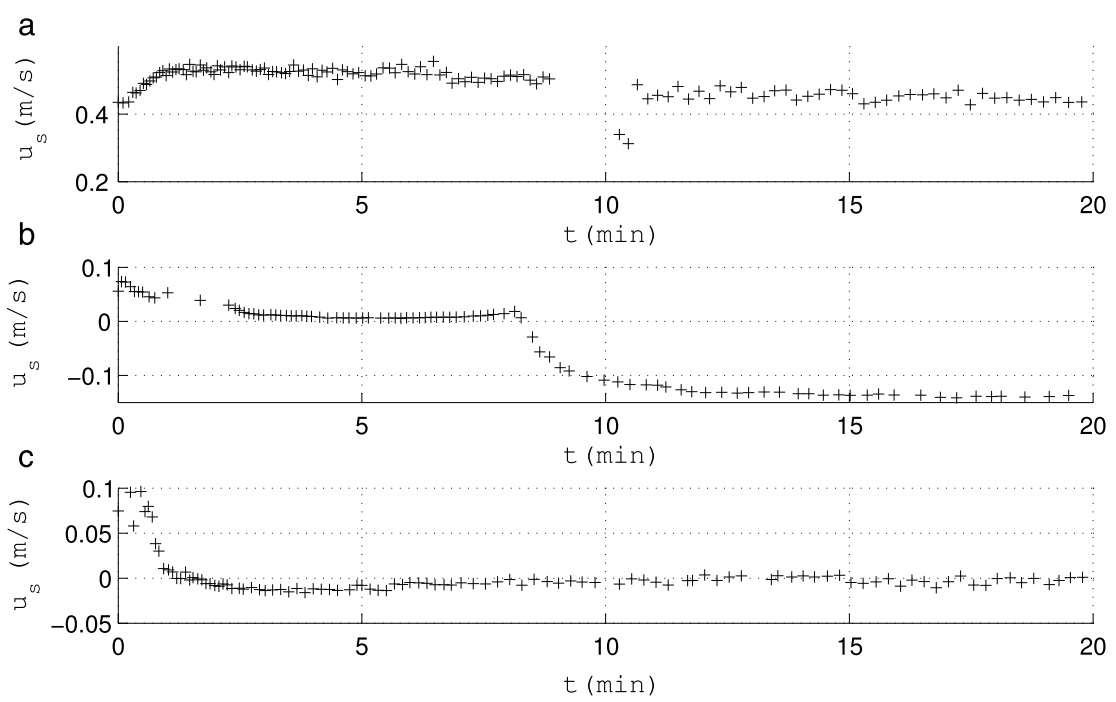

Fig. 7. Time evolution in the fast streaming case $E_{2}$. Axial streaming velocity at (a) $I$ (b) $O_{c-\exp }$ and (c) $O_{e}$. 
the streaming flow is conducted below. Our approach is similar to that developed by Boufermel et al. [24] for computing the average mass transport velocity $\frac{\bar{\rho} \mathbf{v}}{\bar{\rho}}$ in the slow streaming regime, as well as to that developed by Moudjed et al. [31] in the somehow different configuration of bulk streaming.

Focus is on outer streaming, whose transient and established regimes was shown to be drastically modified in the fast nonlinear regime.

\subsection{Equations governing the streaming flow}

Particle velocity $\mathbf{v}$, fluid density $\rho$ and pressure $p$ are decomposed into a fluctuating, periodic component, and a steady component (corresponding to the time average of the variable over the period, i.e. corresponding to the streaming flow)

$$
(\mathbf{v}, \rho, p)=\left(\mathbf{v}^{\prime}+\overline{\mathbf{v}}, \rho^{\prime}+\bar{\rho}, p^{\prime}+\bar{p}\right),
$$

the fluctuating component being denoted by a' superscript and the average operator by an overline. The time average of the fluctuating component is zero. Conversely to usual models of slow streaming, in the present approach no assumption is done on the magnitude of the fluctuation with respect to the steady component. The equations are therefore correct whether the streaming flow is slow or fast.

With this decomposition, the double and triple averaged products between any three variables $f, g, h$ become:

$$
\left\{\begin{array}{l}
\overline{f g}=\bar{f} \bar{g}+\overline{f^{\prime} g h}=\bar{f} \bar{g} \bar{h}+\overline{f^{\prime} g^{\prime}} \bar{h}+\overline{f^{\prime} h^{\prime}} \bar{g}+\overline{g^{\prime} h^{\prime} f}+\overline{f^{\prime} g^{\prime} h^{\prime}} .
\end{array}\right.
$$

The equations governing the streaming flow are obtained by averaging over the acoustic period the full compressible (isentropic) instantaneous Navier-Stokes equations (1) and the isentropic state law (2). Using the double and triple averaged products detailed in Eq. (4) then results in the following time averaged equation system:

$$
\begin{cases}\frac{\partial \bar{\rho}}{\partial t}+\nabla \cdot(\bar{\rho} \overline{\mathbf{v}}) & =-\nabla \cdot\left(\overline{\rho^{\prime} \mathbf{v}^{\prime}}\right) \\ \frac{\partial \bar{\rho} \overline{\mathbf{v}}}{\partial t}+\nabla \cdot(\bar{\rho} \overline{\mathbf{v}} \otimes \overline{\mathbf{v}})+\nabla \bar{p} & =\nabla \cdot(\overline{\bar{\tau}})+\bar{F},\end{cases}
$$

and $\bar{p}-p_{0}=c_{0}^{2}\left(\bar{\rho}-\rho_{0}\right)$, where $\overline{\bar{\tau}}$ is the averaged viscous tensor and $\bar{F}$ is the excitation force given by:

$$
\bar{F}=-\nabla \cdot\left(\overline{\rho^{\prime} \mathbf{v}^{\prime}} \otimes \overline{\mathbf{v}}+\overline{\mathbf{v}} \otimes \overline{\rho^{\prime} \mathbf{v}^{\prime}}+\bar{\rho} \overline{\mathbf{v}^{\prime} \otimes \mathbf{v}^{\prime}}+\overline{\rho^{\prime} \mathbf{v}^{\prime} \otimes \mathbf{v}^{\prime}}\right) .
$$

Note that $\bar{F}$ is the sum of the source terms, representing the nonlinear contribution of the imposed acoustic field.

\subsection{Scale analysis}

The equation system (5) is written in dimensionless form, using standard fluid mechanics scalings, detailed in the following. As stated previously, focus is on the resonator core. In a cylindrical wave guide, streaming cells are toroidal of axial dimension $\lambda / 4$ and radial dimension $R$ in the core of the resonator. Therefore the axial variable $z$ is scaled with $\lambda / 4$ and the radial variable $r$ with $R$.

Mean velocity is scaled with a reference value of the axial streaming velocity $U_{s}$. Density is scaled with $\rho_{0}$. Pressure variations are scaled with $(\delta p)$, not specified at this stage of the scale analysis. The aim of the study is to determine the role of inertia on streaming in the fast regime. Therefore, time is scaled with the characteristic convection time scale in the axial direction $t_{c}=\lambda /\left(4 U_{s}\right)$.

Scalings can then be summarized as follows:

$$
r^{*}=\frac{r}{R}, \quad z^{*}=\frac{z}{\lambda / 4}, \quad \mathbf{v}^{*}=\frac{\overline{\mathbf{v}}}{U_{s}}, \quad t^{*}=\frac{t}{t_{c}}, \quad \rho^{*}=\frac{\bar{\rho}}{\rho_{0}}, \quad p^{*}=\frac{\bar{p}-p_{0}}{(\delta p)} .
$$

Also, the fluctuation variables required to assemble the source terms are scaled with the acoustic amplitudes as follows:

$$
\mathbf{v}^{*}=\frac{\mathbf{v}^{\prime}}{U_{\max }}, \quad \rho^{*}=\frac{\rho^{\prime}}{\rho_{\max }},
$$

$\rho_{\max }$ being the amplitude of density fluctuations at acoustic antinode position in the standing wave. Whenever two different spatial derivatives are involved, the coefficient multiplying each dimensionless term is chosen with respect to the dominant spatial derivative under plane wave assumption: $R \ll \lambda$. In particular, scaling the viscous stress tensor yields a $\frac{\mu U_{s}}{R^{2}}$ factor, and scaling the divergence operator $\nabla \cdot\left(\mathbf{v}^{*}\right)$ yields a $\frac{U_{s}}{\lambda / 4}$ coefficient. The following dimensionless equations are deduced from (5):

$$
\left\{\begin{array}{l}
\frac{\rho_{0}}{t_{c}} \frac{\partial \rho^{*}}{\partial t^{*}}+\frac{\rho_{0} U_{s}}{\lambda / 4} \nabla \cdot\left(\rho^{*} \mathbf{v}^{*}\right)=-\frac{\rho_{\max } U_{\max }}{\lambda / 4} \nabla \cdot\left(\overline{\rho^{\prime *} \mathbf{v}^{\prime *}}\right) \\
\frac{\rho_{0} U_{s}}{t_{c}} \frac{\partial \rho^{*} \mathbf{v}^{*}}{\partial t_{c}^{*}}+\frac{\rho_{0} U_{s}^{2}}{\lambda / 4} \nabla \cdot\left(\rho^{*} \mathbf{v}^{*} \otimes \mathbf{v}^{*}\right)+\frac{(\delta p)}{\lambda / 4} \nabla p^{*}=\frac{\mu U_{s}}{R^{2}} \nabla \cdot\left(\overline{\bar{\tau}}^{*}\right)+\bar{F}
\end{array}\right.
$$


with

$$
\bar{F}=-\frac{\rho_{\max } U_{\max } U_{s}}{\lambda / 4} \nabla \cdot\left(\overline{\rho^{\prime *} \mathbf{v}^{\prime *}} \otimes \mathbf{v}^{*}+\mathbf{v}^{*} \otimes \overline{\rho^{\prime *} \mathbf{v}^{\prime *}}+\frac{\rho_{0} U_{\max }}{\rho_{\max } U_{s}} \overline{\rho^{*}} \overline{\mathbf{v}^{\prime *} \otimes \mathbf{v}^{\prime *}}+\frac{U_{\max }}{U_{s}} \overline{\rho^{\prime *} \mathbf{v}^{\prime *} \otimes \mathbf{v}^{\prime *}}\right) .
$$

Then, multiplying the mass equation with $\lambda /\left(4 \rho_{0} U_{s}\right)$ and the momentum equation with $\lambda /\left(4 \rho_{0} U_{s}^{2}\right)$ and using $t_{c}=\lambda /\left(4 U_{s}\right)$ one can obtain:

$$
\left\{\begin{array}{cc}
\frac{\partial \rho^{*}}{\partial t^{*}}+\nabla \cdot\left(\rho^{*} \mathbf{v}^{*}\right) & =-\frac{\rho_{\max }}{\rho_{0}} \frac{U_{\max }}{U_{s}} \nabla \cdot\left(\overline{\rho^{* *} \mathbf{v}^{* *}}\right) \\
\frac{\partial \rho^{*} \mathbf{v}^{*}}{\partial t^{*}}+\nabla \cdot\left(\rho^{*} \mathbf{v}^{*} \otimes \mathbf{v}^{*}\right)+\frac{(\delta p)}{\rho_{0} U_{s}^{2}} \nabla p^{*} & =\frac{1}{R e} \nabla \cdot\left(\overline{\bar{\tau}}^{*}\right)+\bar{F}^{*}
\end{array}\right.
$$

where the dimensionless excitation force, gathering all the source terms is expressed as:

$$
\begin{aligned}
\bar{F}^{*}= & -\frac{\rho_{\max }}{\rho_{0}} \frac{U_{\max }}{U_{s}} \nabla \cdot\left(\overline{\rho^{\prime *} \mathbf{v}^{\prime *}} \otimes \mathbf{v}^{*}+\mathbf{v}^{*} \otimes \overline{\rho^{\prime *} \mathbf{v}^{\prime *}}\right) \\
& -\frac{U_{\max }^{2}}{U_{s}^{2}} \nabla \cdot\left(\rho^{*} \overline{\mathbf{v}^{\prime *} \otimes \mathbf{v}^{\prime *}}\right)-\frac{\rho_{\max }}{\rho_{0}} \frac{U_{\max }^{2}}{U_{s}^{2}} \nabla \cdot\left(\overline{\rho^{\prime *} \mathbf{v}^{\prime *} \otimes \mathbf{v}^{\prime *}}\right) .
\end{aligned}
$$

The system equations (Eq. (11)) is the dimensionless form of the general governing streaming equations (Eq. (5)). The Reynolds number $R e=\frac{4 U_{s}}{\lambda} \frac{R^{2}}{v}$ that appears in the momentum equation is the Reynolds number characterizing the mean flow in a thin tube, defined as the ratio between the diffusion time scale in the radial direction $t_{d}=R^{2} / v$ and the convection time scale in the axial direction $t_{c}=\lambda /\left(4 U_{s}\right), R e=t_{d} / t_{c}$. Note that $t_{d}$ is equivalent (with a different length reference value) to the characteristic time for streaming development defined by Amari et al. in [32] and used to obtain the transient linear streaming equations. Under the assumptions that acoustic perturbations are small (i.e. of order $M=\frac{U_{\max }}{c_{0}} \ll 1$ ) and the streaming field is of order $M^{2}\left(U_{s}=M U_{\max }\right)$, this Reynolds number can be rewritten as $R e=\frac{4}{\pi} \frac{M^{2}}{S h^{2}}=\frac{4}{\pi} R e_{N L}, R e_{N L}$ being the commonly introduced nonlinear Reynolds number.

Under these assumptions, the Reynolds number $R e$ and the nonlinear Reynolds number $R e_{N L}$ are thus of the same order of magnitude. However, since the Reynolds number Re is defined directly with the value of the maximum streaming velocity, it describes more accurately the influence of inertia in the nonlinear regime in the core of the wave guide than $R e_{N L}$. Indeed, previous numerical and experimental studies [29] have shown that in the nonlinear regime, the maximum value of the axial streaming velocity on the tube axis is much smaller than slow streaming expectations $3 U_{\max }^{2} /(8 c)$. Therefore the nonlinear Reynolds number $R e_{N L}$ overestimates the influence of inertia on the streaming flow. For example, in the numerical case $N_{2}$, the Reynolds number based on the maximum value of $U_{S}$ on the axis is $R e=17.1$ while the corresponding nonlinear Reynolds number is $R e_{N L}=80.84$. Nonetheless $R e$ cannot be estimated before the simulations/experiments whereas $R e_{N L}$ can be calculated from the acoustics and thus remains useful.

Another Reynolds number used in the literature to distinguish linear from nonlinear regime was the streaming Reynolds number used by Stuart et al. [11] defined as $R e_{s}=\frac{U_{s} \lambda}{2 \pi v}$. It can be expressed as $R e_{s}=R e \frac{\lambda^{2}}{R^{2}}$ and also has the drawback to overestimate the nonlinear inertial effects on Rayleigh streaming.

In the case $R e \ll 1$ (which also means that convection time is much greater than diffusion time) and under the assumption of small perturbations $\left(M=\frac{U_{\max }}{c_{0}}=\frac{U_{s}}{U_{\max }} \ll 1\right)$, the inertial term and the time depending term can both be neglected with respect to the viscous term. The magnitude of source terms is then estimated :

$$
\begin{aligned}
& \frac{\rho_{a c}}{\rho_{0}} \frac{U_{a c}}{U_{s}} \nabla \cdot\left(\overline{\rho^{\prime *} \mathbf{v}^{*}} \otimes \mathbf{v}^{*}+\mathbf{v}^{*} \otimes \overline{\rho^{\prime *} \mathbf{v}^{\prime *}}\right)=O\left(M \cdot \frac{1}{M}\right)=O(1), \\
& \frac{U_{\max }^{2}}{U_{s}^{2}} \nabla \cdot\left(\rho^{*} \overline{\mathbf{v}^{\prime *} \otimes \mathbf{v}^{\prime *}}\right)=O\left(\frac{1}{M^{2}}\right), \\
& \frac{\rho_{\max }}{\rho_{0}} \frac{U_{\max }^{2}}{U_{s}^{2}} \nabla \cdot\left(\overline{\rho^{\prime *} \mathbf{v}^{\prime *} \otimes \mathbf{v}^{\prime *}}\right)=O\left(M \cdot \frac{1}{M^{2}}\right)=O\left(\frac{1}{M}\right) .
\end{aligned}
$$

$\bar{F}^{*}$ is then of order $\frac{1}{M^{2}}$. The pressure gradient must have the same order of magnitude as $\bar{F}^{*}$ which yields also the pressure variations scale $(\delta p)=\rho_{0} U_{\max }^{2}=p_{0} M^{2}$. The magnitude of the source term in the mass equation is estimated as previously: $\frac{\rho_{\max }}{\rho_{0}} \frac{U_{\max }}{U_{s}} \nabla \cdot\left(\overline{\rho^{\prime *} \mathbf{v}^{\prime *}}\right)=O(1)$. Finally, the steady linear equations for the streaming flow (dimensional form) are then deduced from the first order approximation of the momentum equation and (5) gives:

$$
\left\{\begin{aligned}
\nabla \cdot(\bar{\rho} \overline{\mathbf{v}}) & =-\nabla \cdot\left(\overline{\rho^{\prime} \mathbf{v}^{\prime}}\right) \\
\nabla \bar{p} & =\nabla \cdot(\overline{\bar{\tau}})-\overline{\mathbf{F}}
\end{aligned}\right.
$$

where $\bar{F} \approx \nabla \cdot\left(\bar{\rho} \overline{\mathbf{v}^{\prime} \otimes \mathbf{v}^{\prime}}\right)$.

As the $R e$ number is increased i.e. $R e \gtrsim 1$, inertial and time depending terms cannot be neglected anymore and the complete unsteady form of streaming equations (5) with all the source terms should be used. In this case the diffusion time scale becomes larger than the convection time scale, thus the overall process is faster and the steady state is reached earlier. 
Table 2

Reynolds numbers of different presented cases ( $N$ : numerical, $E$ : experimental).

\begin{tabular}{llllll}
\hline Name & $U_{s}($ at $I)(\mathrm{m} / \mathrm{s})$ & $U_{s}\left(\right.$ at $\left.O_{c}\right)(\mathrm{m} / \mathrm{s})$ & $\operatorname{Re} e_{N L}$ & $R e(I)$ \\
\hline$N_{1}$ & $2.42 \times 10^{-4}$ & $2.63 \times 10^{-4}$ & 0.005 & $2.24 \times 10^{-3}$ \\
$N_{2}$ & 3.18 & 1.84 & 80.84 & $2.43 \times 10^{-3}$ \\
$E_{2}$ & 0.5 & 0.01 & 85.22 & 17 & 29.44 \\
\hline
\end{tabular}

\subsection{Reynolds numbers and time scale values for slow and fast outer streaming}

Table 2 displays the values of the axial streaming velocity at points $I$ or $O_{c}$, and of the corresponding Reynolds number of previously described cases $N_{1}, N_{2}$ and $E_{2}$.

The previous analysis can also be used to estimate quantitatively the expected time scales of the streaming time evolutions for outer streaming, in the slow and fast streaming regime, that was described qualitatively in Section 3.

In the slow streaming case, diffusion of momentum in the radial direction is expected to be dominant. The convection time variations take place over a very long scale and they are very small, therefore only time variations over a diffusion time scale are significant. The associated characteristic time scale for the establishment of outer streaming is $t_{d}=R^{2} / \nu$, which, using the parameter values in Table 2 for $N_{1}$, amounts to $t_{d}=0.026 \mathrm{~s}$. Taking into account the fact that the streaming velocity at $O_{c}$ in Fig. 4(b) is not established in $0.01 \mathrm{~s}$, this is a coherent result.

Based on known analytical solutions of the unsteady pure diffusion equation, if diffusion were dominant the time evolution curves should be in the form $u(t)=u_{\infty}\left(1-e^{-t / \tau}\right)$, where the time constant $\tau$ is the time needed for the value $u$ to reach $63 \%$ of the established value $u_{\infty}$ and the time constant needed to achieve $99.999 \%$ of the established value is equal to $10 \tau$. The time evolution curve at $O_{c}$ in Fig. 4(b) is actually very well fitted with such a diffusion curve, and the quantitative fit gives $\tau \simeq 0.0015 \mathrm{~s}$ and therefore $10 \tau=0.015 \mathrm{~s}$, which is the same order of magnitude as $t_{d}$.

In the fast streaming case $N_{2}$, in the core, transverse diffusion is expected to compete with convection in the axial direction. The value of the theoretical diffusion time scale $t_{d}$ is still the same, since it does not depend on the excitation level $\left(t_{d}=0.026 \mathrm{~s}\right)$. The first velocity increase in the time evolution curve at $O_{c}$ (Fig. 6(b)) can be fitted by assuming dominant diffusion and the obtained characteristic time scale is the same as in the slow streaming case. Therefore transverse diffusion is dominant at first in the establishment of the outer streaming, just like in the slow streaming case. Assuming next that the second velocity increase in the time evolution curve at $O_{c}$ (Fig. $6(\mathrm{~b})$ ) is related to convection, the theoretical time scale associated with convection along the length $\lambda / 4$ with velocity $U_{s}$ at the location $I$ is $t_{c}=\lambda /\left(4 U_{s}\right)=1.6 \times 10^{-3} \mathrm{~s}$ based on the parameters in Table 2. This increase is 20 times faster than that of the diffusion process. A linear fit of the second velocity increase at $O_{c}$ on Fig. 6(b) gives $t_{c f i t} \approx 10^{-3} \mathrm{~s}$, which is of the same order as $t_{c}$. This shows that the second velocity increase is indeed related to convection in the axial direction. Note that convective transport is much faster than diffusive transport and that the steady state is reached earlier than in the slow streaming case $N_{1}$, in agreement with the analysis given at the end of Section 4.2.

In the experimental case E2, Fig. 7 shows the evolution of streaming at the three different locations $I, O_{c-\exp }$ and $O_{e}$. Two separate slopes in the time evolution of streaming at $O_{c-\exp }$ are also observed, but these are not due to the competition between diffusion and convection as was the case for numerical results. Actually our experimental set up does not allow to observe this competition: the theoretical value of the radial diffusion time scale is $t_{d}=24 \mathrm{~s}$ and the convection time scale in the axial direction calculated for $E_{2}$ based on the maximum value of $U_{s}$ at $I$ location (read in Fig. $7(\mathrm{a})$ ) is $t_{c E 2}=0.71 \mathrm{~s}$. It can be observed that acoustics requires about $3 \mathrm{~min}$ to be established. The transient of acoustics is therefore too long to allow to separately identify the establishment of streaming. Moreover one measurement point actually corresponds to an integration of results of particle velocity measurements over several seconds: indeed in order to obtain converged results of the particle velocity estimate, several thousands of seeding particles need to have crossed the measuring volume [27,33]. The associated measuring time depends on the data rate associated with the seeding density; in our set up this process can take as long as $10 \mathrm{~s}$. Therefore it is impossible to distinguish time scales as short as $t_{d}$ or $t_{c}$. The evolution of streaming shown in Fig. 7 can be supposed to result from heat transfer effects. Indeed measurements give an estimate of the mean velocity, that is equal to streaming velocity only if no heat convection occurs. In the case of $E_{2}$ experiments, a $4 \mathrm{~K}$ temperature difference in the guide wall temperature was measured across a distance of $\lambda / 4$. The temperature establishment was found to last for 20 min, probably due to the time needed to reach thermal equilibrium between thermoacoustic, conduction and convection heat fluxes. It can thus be assumed that the measured time evolution of mean velocity is not the only result of acoustic streaming. In the following, only the numerical results will be considered.

In the next section, the driving effect of inner streaming on the outer streaming will be analyzed. Indeed, an analogy with the lid-driven cavity in a cylindrical geometry is presented in order to identify the physical meanings of the characteristic times.

\section{Lid-driven cavity analogy}

Based on the observation that inner streaming drives outer streaming with competing diffusion and convection, and in order to mimic the establishment of outer streaming, we introduce an equivalent fluid-mechanics problem. We therefore consider the establishment of flow in a lid-driven cavity, but with a cylindrical geometry, and assume that the "lid" is the tube envelope where the inner streaming velocity is imposed. 


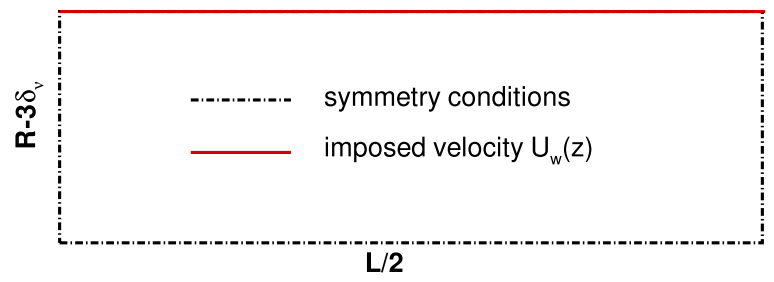

Fig. 8. Schematics of the lid-driven cylindrical flow problem: geometry and boundary conditions.
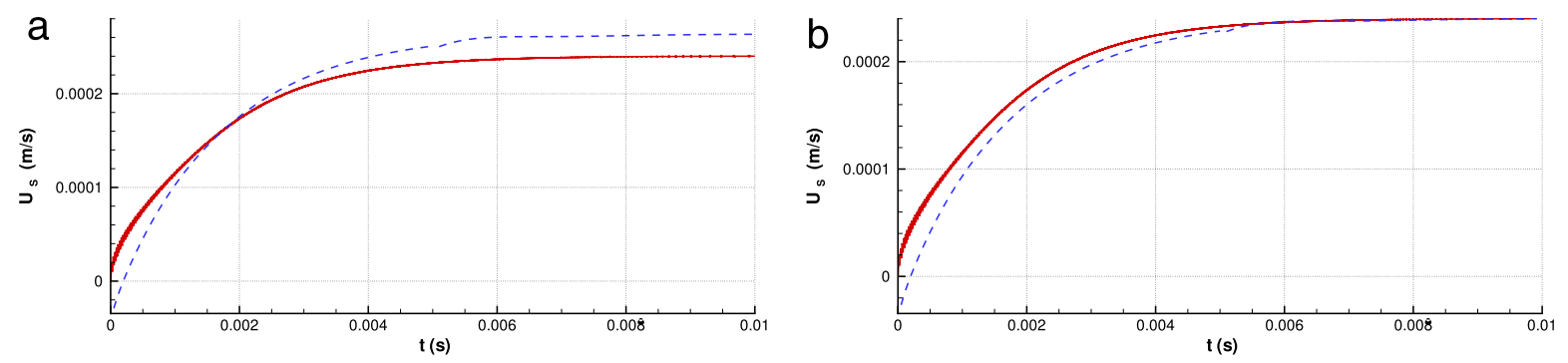

Fig. 9. (a) Time evolution ( - ) of $U(z=L / 4, r=0)$ in lid-driven cavity for $U_{w}$ from $N_{1}$, and ( - - ) of $U_{s}$ at $O_{c}$ from Fig. 4. (b) Same with both curves normalized to reach the same steady-state value. (For interpretation of the references to color in this figure legend, the reader is referred to the web version of this article.)

\subsection{Description of the problem}

The problem being axisymmetric, the geometry is defined by $z \in[0, L / 2]$ and $r \in\left[0, R-3 \delta_{v}\right]$. The viscous flow is governed by the following equations, which are the same as in Eq. (1) without the acoustic source terms:

$$
\left\{\begin{array}{l}
\frac{\partial \rho}{\partial t}+\nabla \cdot(\rho \mathbf{v}) \quad=0 \\
\frac{\partial \rho \mathbf{v}}{\partial t}+\nabla \cdot(\rho \mathbf{v} \otimes \mathbf{v})+\nabla p=\nabla \cdot(\overline{\bar{\tau}})
\end{array}\right.
$$

This system is completed with the isentropic state law Eq. (2). Symmetry conditions are imposed at the vertical walls $z=0, L / 2$ and on the horizontal axis $r=0$. The boundary condition at the wall $r=R-3 \delta_{v}$ (the "lid") is that the velocity is only axial. Its value $U_{w}(z)$ is equal to the streaming axial velocity profile $U_{s}(z)$ along the tube at $r=R-3 \delta_{v}$ given by direct numerical calculations described in the previous sections (see Fig. 8). There is initially no flow in the cavity. The numerical procedure is the same as that described in Section 2.2. The simulations show that the flow is almost incompressible (density variations are less than $0.006 \%$ ).

\subsection{Results}

A first simulation (low level) is run with $U_{w}$ corresponding to the established $U_{s}$ at $r=R-3 \delta_{v}$ for $N_{1}$ case (slow streaming). Fig. 9(a) shows (in red) the time evolution of the axial velocity $U$ at $r=0$ and $z=L / 4$ (center of the mimicked streaming cell). The time evolution of the streaming velocity at $O_{c}$ (same location) in Fig. 4 is reproduced in blue dots. Fig. 9 (b) shows both curves normalized so that they reach the same steady state value. Both curves are very similar with similar time scales, which confirms that transverse diffusion of momentum is the dominant physical phenomenon for the establishment of the slow streaming pattern.

A simulation at higher level is run with $U_{w}$ corresponding to the established $U_{s}$ at $r=R-3 \delta_{v}$ for $N_{2}$ case (fast streaming). Fig. 10(a) shows (solid red line) the time evolution in the lid-driven cavity of the axial velocity $U$ at $r=0$ and $z=L / 4$. The time evolution of the streaming velocity at $O_{c}$ (same location) given by Fig. $6(\mathrm{~b})$ is reproduced in blue dots. Both curves are very similar in shape, although the maximum value obtained for the streaming curve is about half of that in the lid-driven cavity. Fig. 10(b) shows the same curves normalized to reach the same steady-state. Both curves are almost superimposed, which shows that the time scales are the same and that the change of slope in the curve is indeed related to the inertial effect, when convection becomes strong enough to compete efficiently with diffusion and to modify the time evolution. However, as stated above the established streaming velocity is much smaller than that obtained in the lid-driven cavity. This suggests that the inertial effects of the lid-driven cavity model are not sufficient to correctly model the change in the streaming flow field.

Fig. 11 shows several snapshots of contours of the axial velocity in the lid-driven cavity during the transient evolution. Streamlines are superimposed on the right half plane section (the simulations are performed on a length $L / 2$ and the solution 

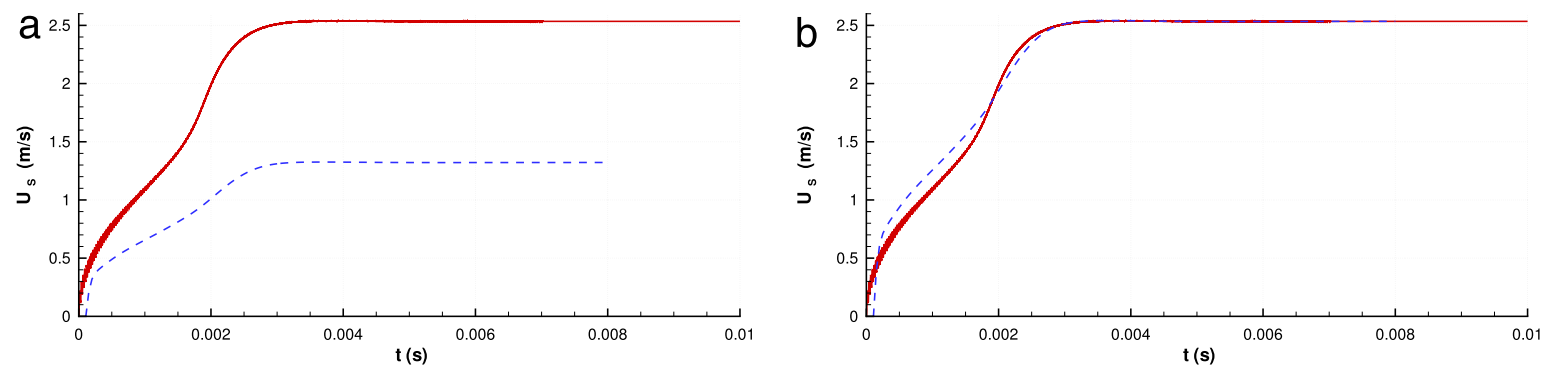

Fig. 10. (a) Time evolution ( -$)$ of $U(z=L / 4, r=0)$ in lid-driven cavity for $U_{w}$ from $N_{2}$, and $(--)$ of $U_{s}$ at $O_{c}$ from Fig. 6. (b) Same with both curves normalized to reach the same steady-state value. (For interpretation of the references to color in this figure legend, the reader is referred to the web version of this article.)

in the right half plane section is constructed by symmetry). Comparison with Fig. 5 shows that the time evolution of patterns are very similar which confirms that the transient evolution of the streaming patterns comes from competing diffusion and convection in a manner analogous to the lid-driven cavity. However, an important difference exists: there is no extra streaming cell in the lid-driven cavity.

Fig. 12 shows axial profiles of $U$ on the axis $r=0$ for corresponding times of Fig. 11. The axial profile imposed at the wall $r=R-3 \delta_{v}$ is also shown. At early times the axial profile of $U$ is similar to the profile of $U_{w}$, which confirms that momentum diffusion in the radial direction is dominant. The distortion observed in the last profile is due to convection in the axial direction.

A similar simulation can be conducted using the experimental configuration of $E_{2}$. Fig. 13 shows the time evolution of the axial velocity $U$ at $r=0$ and $z=L / 4$ in the lid-driven cavity (the experimental inner streaming axial profile is imposed at the lid). The associated time scales are of the same order as $t_{d}$ and $t_{c}$ calculated in Section 4.3, and the transient behavior of the flow is analogous to that observed in Fig. 10. As mentioned earlier, this evolution cannot be highlighted experimentally.

This analysis shows that although diffusion and convection do compete in the establishment of the streaming flow in a similar manner as in the lid-driven cavity problem, this is not enough to explain the emergence of the new streaming cell. Therefore, at this stage, we can conclude that the lid-cavity model is not complete enough to describe the mutation of streaming at high levels.

In the next section, the complete streaming equations (5) are solved numerically, with a mono-frequency acoustic source used to compute the different source terms. The effect of increasing the amplitude of the acoustic flow field is analyzed.

\section{Averaged model simulation for the streaming flow}

The high-level mono-frequency acoustic field source used in this section is obtained in two steps. Firstly a DNS simulation at very low-level amplitude is run until a steady streaming flow is obtained. The resulting acoustic field, calculated by subtracting the streaming flow from the total velocity field $\left(\mathbf{v}^{\prime}=\mathbf{v}-\overline{\mathbf{v}}, \rho^{\prime}=\rho-\bar{\rho}\right)$, contains insignificant level of harmonics. Then this acoustic field $\left(\mathbf{v}^{\prime}, \rho^{\prime}\right)$ is multiplied by a coefficient $\alpha$ chosen to obtain high values of the nonlinear Reynolds number $R e_{N L}$. This acoustic field is used to compute the source terms so that the following averaged Navier-Stokes equations are solved for the unknown streaming field $\left(\mathbf{v}_{S}, \rho_{S}, p_{S}\right)$ :

$$
\begin{cases}\frac{\partial \rho_{S}}{\partial t}+\nabla \cdot\left(\rho_{S} \mathbf{v}_{S}\right) & =-\alpha^{2} \nabla \cdot\left(\overline{\rho^{\prime} \mathbf{v}^{\prime}}\right) \\ \frac{\partial \rho_{S} \mathbf{v}_{S}}{\partial t}+\nabla \cdot\left(\rho_{S} \mathbf{v}_{S} \otimes \mathbf{v}_{S}\right)+\nabla p_{S} & =\nabla \cdot\left(\overline{\bar{\tau}}_{S}\right)+\bar{F}_{1},\end{cases}
$$

with $\bar{F}_{1}$ defined by

$$
\bar{F}_{1}=-\nabla \cdot\left(\alpha^{2} \overline{\rho^{\prime} \mathbf{v}^{\prime}} \otimes \mathbf{v}_{S}+\alpha^{2} \mathbf{v}_{S} \otimes \overline{\rho^{\prime} \mathbf{v}^{\prime}}+\alpha^{2} \rho_{S} \overline{\mathbf{v}^{\prime} \otimes \mathbf{v}^{\prime}}+\alpha^{3} \overline{\rho^{\prime} \mathbf{v}^{\prime} \otimes \mathbf{v}^{\prime}}\right)
$$

and $p_{S}-p_{0}=c_{0}^{2}\left(\rho_{S}-\rho_{0}\right)$. The boundary conditions are no slip on the outside wall parallel to the horizontal symmetry axis, and symmetry conditions on the vertical boundaries and horizontal axis.

These equations, very similar to Eqs. (5), are solved with the same numerical method as presented in Section 2.2.

This AMS approach can be used to study the influence of inertial effects on the streaming flow for a monofrequency high amplitude acoustic forcing field. This is equivalent to the strategy followed in [15]. In this way we are able to exclude the influence on the streaming flow of higher harmonics in the acoustic signal. This numerical approach is used here to investigate nonlinear Reynolds numbers $R e_{N L}$ ranging from 0.002 up to 94 (corresponding to $R e$ ranging from 0.0025 to 30.81).

Fig. 14 displays the axial streaming velocity component $U_{S}$ obtained with the AMS simulation scaled by the Rayleigh streaming maximum velocity $3 \times U_{\max }^{2} /\left(8 c_{0}\right)$ along the scaled central axis $z / L$ for $R / \delta_{v}=40$ and $0.002 \leq R e_{N L} \leq 94$. For 


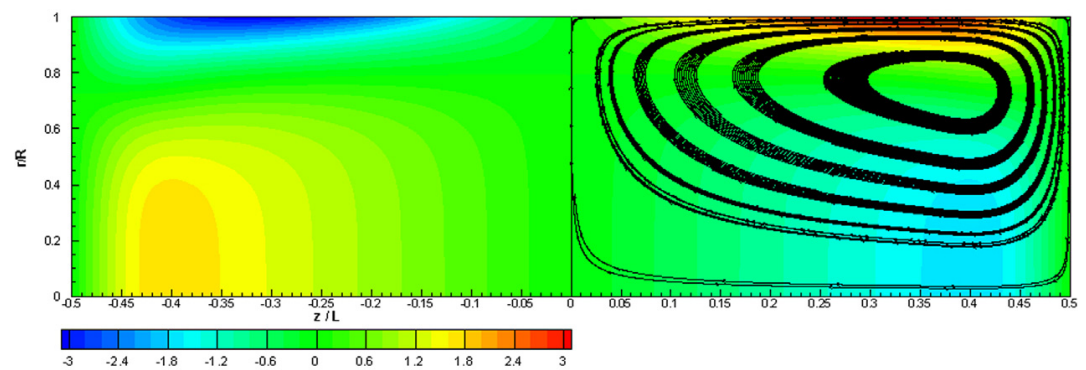

(a) $t=7.5 \times 10^{-4} \mathrm{~s}$.

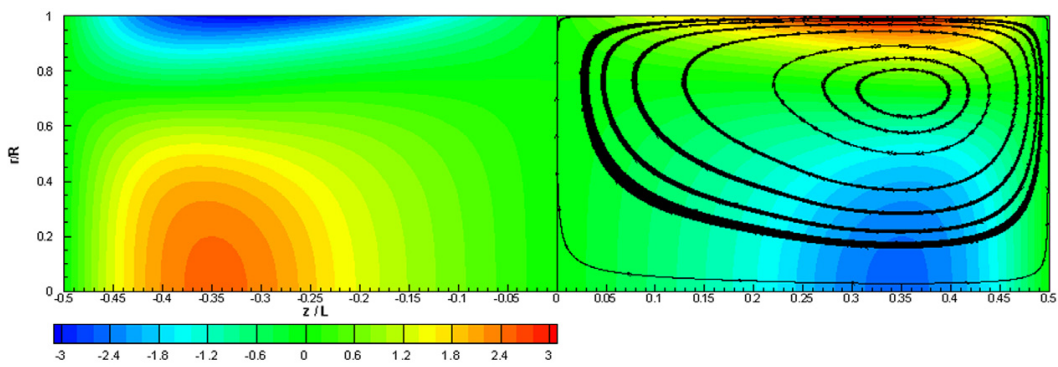

(b) $t=1.25 \times 10^{-3} \mathrm{~s}$

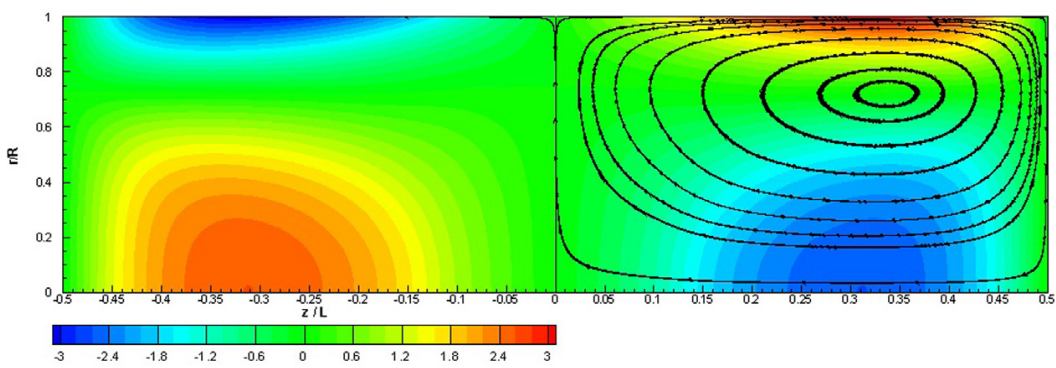

(c) $t=1.75 \times 10^{-3} \mathrm{~s}$.

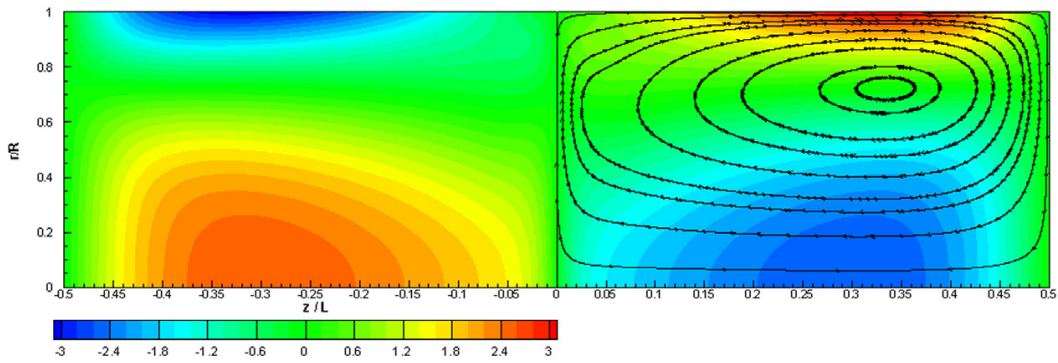

(d) $t=1 \times 10^{-2} \mathrm{~s}$.

Fig. 11. Contours of the axial velocity (with color code) at different times during the transient evolution in the lid-driven cavity for $U_{w}$ from $N_{2}$. Streamlines are superimposed on the right half plane section. (For interpretation of the references to color in this figure legend, the reader is referred to the web version of this article.)

increasing $R e_{N L}$ numbers, the sine function associated to slow streaming becomes steeper next to the resonator center, at the acoustic velocity antinode location, and not next to the resonator ends (acoustic velocity nodes locations). This behavior is in agreement with the predictions of the nonlinear model developed by Menguy and Gilbert [15] at moderate nonlinear Reynolds numbers $R e_{N L}=O(1)$. The direction of distortion is opposite to that observed in the full DNS simulations and experiments [28,29].

In Fig. 15, the dimensionless streaming velocity is plotted along the axis for DNS, AMS and Lid-driven cavity (LDC) simulations, for slow-streaming $\left(N_{1}\right)$ and fast-streaming $\left(N_{2}\right)$. For slow-streaming, all three simulations give analogous 


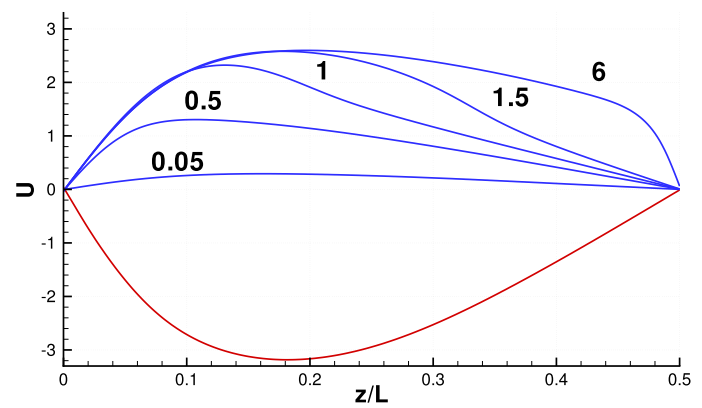

Fig. 12. Axial profiles of $U$ at $r=0$ (in blue) for corresponding times expressed in $10^{-3} \mathrm{~s}$ and at $r=R-3 \delta_{v}$ (in red) in the lid-driven cavity for $U_{w}$ from $N_{2}$. (For interpretation of the references to color in this figure legend, the reader is referred to the web version of this article.)

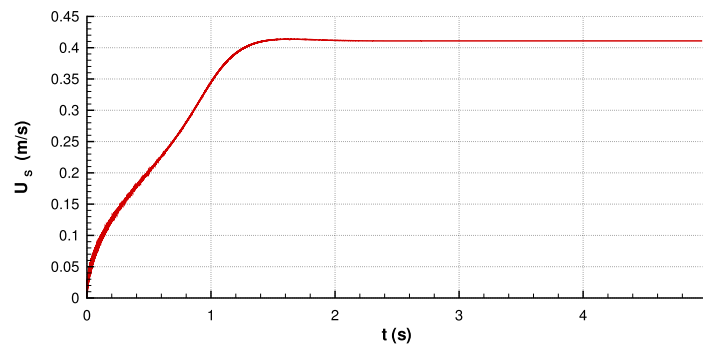

Fig. 13. Time evolution of $U(z=L / 4, r=0)$ in lid-driven cavity for $U_{w}$ from $E_{2}$.

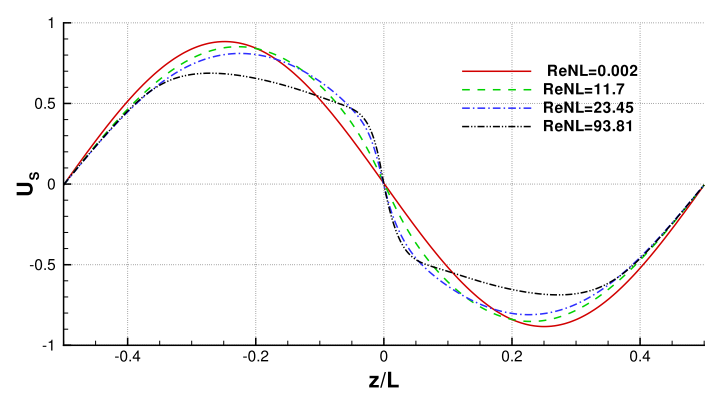

Fig. 14. AMS results for streaming velocity scaled by $3 \times U_{\max }^{2} /\left(8 c_{0}\right)$ along the axis, $R / \delta_{v}=40$.

profiles even though the LDC streaming velocity is slightly smaller. This is because the lid-cavity model does not include the acoustic source terms.

For fast-streaming, the AMS result shows the same behavior as the lid-driven cavity. Once again the LDC streaming velocity is slightly smaller, because that model does not include the acoustic source terms. For the AMS simulations, the associated axial velocity contours and streamlines are displayed in Fig. 16. They are similar to those obtained for the lid driven cavity flow, in the steady state (see Fig. 11(d)). On the contrary, the DNS streaming velocity on the axis is globally much smaller, and shows the presence of new streaming cells.

Both the lid-driven cavity and the AMS approaches show that when the intensity of a monofrequency wave is increased, the streaming flow becomes nonlinear because of inertial effects, but the distortion of the Rayleigh streaming profile is different than in DNS simulations or experiments.

This shows that in order to explain the mutation of the streaming flow at high levels, other effects must be analyzed. The only effects that have not been considered yet are the consequences of nonlinear interaction between the streaming flow and acoustics, i.e. the modification of the acoustic flow by the streaming flow. This is currently under study.

\section{Conclusion}

In this paper the effect of inertia on Rayleigh streaming was investigated numerically and experimentally.

Time evolution of streaming patterns were analyzed. Based on an analysis of the equations driving the streaming flow, two time scales were identified: the diffusion and the convection time scales. The ratio of these time scales is a Reynolds 


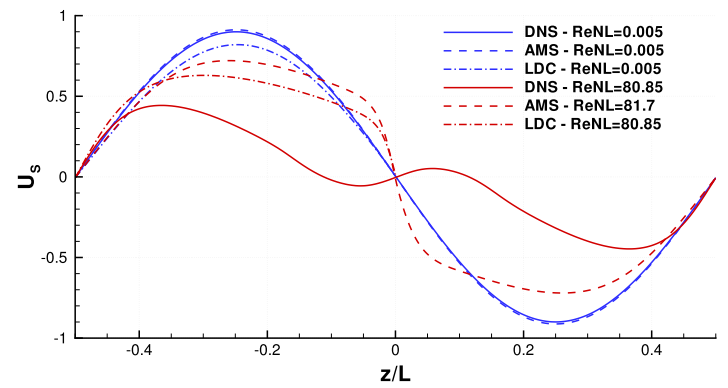

Fig. 15. Dimensionless streaming velocity along the axis. Comparison of DNS (solid lines), AMS (dashed lines) and Lid-Driven Cavity, LDC (dashed-dotted lines) results, $R / \delta_{v}=50$, for slow-streaming (in blue) and fast-streaming (in red). (For interpretation of the references to color in this figure legend, the reader is referred to the web version of this article.)

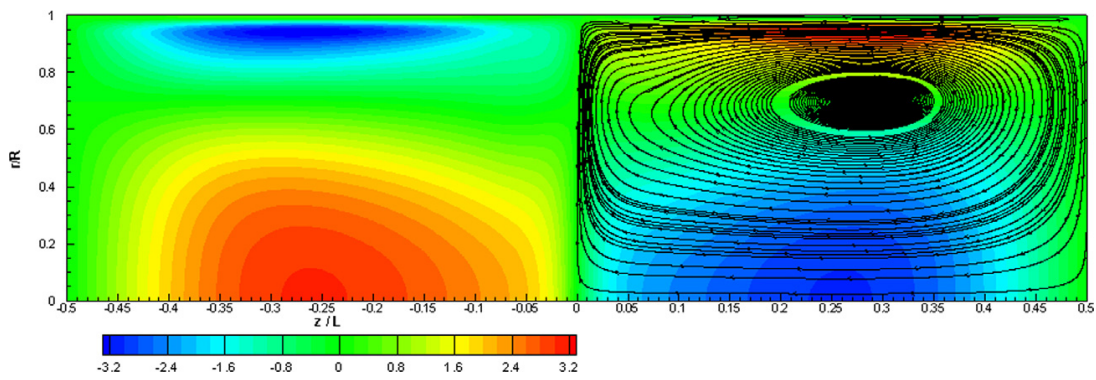

Fig. 16. AMS results, $R / \delta_{v}=50$. Isolines of the axial streaming velocity colored by its value and the streamlines of the streaming flow. (For interpretation of the references to color in this figure legend, the reader is referred to the web version of this article.)

number $R e=\frac{4 U_{s}}{\lambda} \frac{R^{2}}{v}$ that characterizes the streaming flow and separates the linear from the nonlinear regime. This number is proportional to the nonlinear Reynolds number $R e_{N L}$ only when the streaming flow is of order $M^{2} c_{0}$.

The time evolution of outer streaming was mimicked by analyzing a lid-driven flow. It was confirmed that in a first stage, the outer fast streaming cells are created due to momentum diffusion in the radial direction and that in a second stage, the streaming cells are distorted due to convection in the axial direction. But no extra cell is created in the lid-driven cavity.

Nonlinear inertial effects on the established streaming flow were further investigated using a numerical approach that solves the full streaming flow equations with an imposed monofrequency acoustic wave source computed from the DNS. These simulations as well as the lid-driven cavity simulations show similar results: The streaming patterns in the core of the waveguide are different than those observed in DNS simulations and experiments.

Therefore inertial effects cannot be considered as the leading phenomenon to explain the mutation of streaming at high acoustic levels. Nonlinear interactions between the streaming flow and acoustics have to be considered, which will be the scope of further studies.

\section{Acknowledgment}

The authors wish to thank J. Gilbert for many fruitful discussions on this work.

\section{References}

[1] G.W. Swift, D.L. Gardner, S. Backhaus, Acoustic recovery of lost power in pulse tube refrigerators, J. Acoust. Soc. Am. 105 (1999) $711-724$.

[2] L. Rayleigh, On the circulation of air observed in Kundt's tubes, and on some allied acoustical problems, Philos. Trans. R. Soc. 175 (1884) 1-21.

[3] H. Schlichting, Berechnung ebener periodischer Grenzschicht- strommungen [calculation of plane periodic boundary layer streaming], Phys. Zcit. 33 (1932) 327-335.

[4] W.L. Nyborg, Acoustic streaming, in: W.P. Mason (Ed.), Physical Acoustics, Vol. 2B, Academic Press, New York, 1965, pp. 265-331.

[5] P.J. Westervelt, The theory of steady rotational flow generated by sound fields, J. Acoust. Soc. Am. 25 (1953) 60-67.

[6] O.V. Rudenko, S.I. Soluyan, Theoretical Fundations of Non- Linear Acoustics, English Translation, Plenum, New York, 1977, p. 187-211.

[7] N. Rott, The influence of heat conduction on acoustic streaming, Z. Angew. Math. Phys. 25 (1974) 417-421.

[8] Q. Qi, The effect of compressibility on acoustic streaming near a rigid boundary for a plane traveling wave, J. Acoust. Soc. Am. 94 (1993) $1090-1098$.

[9] H. Bailliet, V. Gusev, R. Raspet, R.A. Hiller, Acoustic streaming in closed thermoacoustic devices, J. Acoust. Soc. Am. 110 (2001) $1808-1821$.

[10] M.F. Hamilton, Y.A. Ilinskii, E.A. Zabolotskaya, J. Acoust. Soc. Am. 114 (6) (2003) 3092-3101.

[11] J.T. Stuart, Double boundary layer in oscillatory viscous flow, J. Fluid Mech. 24 (1966) 673-987.

[12] J. Lighthill, Acoustic streaming, J. Sound Vib. 61 (1978) 391-418.

[13] V.E. Gusev, O.V. Rudenko, Nonsteady quasi-one-dimensional acoustic streaming in unbounded volumes with hydrodynamic nonlinearity, Sov. Phys. Acoust. 25 (1979) 493-497. 
[14] N. Riley, Streaming from a cylinder due to an acoustic source, J. Fluid Mech. 180 (1987) 319-326.

[15] L. Menguy, J. Gilbert, Non-linear acoustic streaming accompanying a plane stationary wave in a guide, Acta Acust. 86 (2000) $249-259$.

[16] A. Gopinath, A.F. Mills, Convective heat transfer due to acoustic streaming across the ends of a Kundt tube, J. Heat Transfer 116 (1994) $47-53$.

[17] M. Kawahashi, M. Arakawa, Nonlinear phenomena induced by finite-amplitude oscillation of air column in closed duct, JSME Int. J. 39 (1996) $280-286$.

[18] M.K. Aktas, B. Farouk, Numerical simulation of acoustic streaming generated by finite-amplitude resonant oscillations in an enclosure, J. Acoust. Soc. Am. 116 (5) (2004) 2822-2831.

[19] V. Daru, D. Baltean-Carlès, C. Weisman, P. Debesse, G. Gandikota, Two-dimensional numerical simulations of nonlinear acoustic streaming in standing waves, Wave Motion 50 (2013) 955-963.

[20] A.A. Gubaidullin, A.V. Yakovenko, Effects of heat exchange and nonlinearity on acoustic streaming in a vibrating cylindrical cavity, J. Acoust. Soc. Am. 137 (2015) 3281-3287.

[21] A.A. Gubaidullin, A.V. Pyatkova, Acoustic streaming with allowance for heat transfer, Acoust. Phys. 62 (3) (2016) 300-305.

[22] C. Scalo, S.K. Lele, L. Hesselink, Linear and nonlinear modelling of a theoretical travelling-wave thermoacoustic heat engine, J. Fluid Mech. 766 (2015) 368-404.

[23] M. Cervenka, M. Bednarik, Variety of acoustic streaming in 2D resonant channels, Wave Motion 66 (2016) 21-30

[24] A. Boufermel, N. Joly, P. Lotton, M. Amari, V. Gusev, Velocity of mass transport to model acoustic streaming: Numerical application to annular resonators, Acta Acust. United Acust. 97 (2) (2011) 219-227.

[25] S. Boluriaan, P.J. Morris, Numerical simulation of acoustic streaming in high amplitude standing wave, in: AAIA Paper 2003-3152 (9th AIAA/CEAS Aeroacoustics Conference and Exhibit, 12-14 May 2003, Hilton Head, South Carolina).

[26] M. Thompson, A. Atchley, M. Maccarone, Influences of a temperature gradient and fluid inertia on acoustic streaming in a standing wave, J. Acoust. Soc. Am. 117 (2005) 1839-1849.

[27] S. Moreau, H. Bailliet, J.-C. Valière, Measurements of inner and outer streaming vortices in a standing waveguide using laser doppler velocimetry, J. Acoust. Soc. Am. 123 (2008) 640-664.

[28] I. Reyt, H. Bailliet, J.C. Valière, Experimental investigation of acoustic streaming in a cylindrical wave guide up to high streaming Reynolds numbers, J. Acoust. Soc. Am. 135 (2014) 27-37.

[29] I. Reyt, V. Daru, H. Bailliet, S. Moreau, J.C. Valière, D. Baltean-Carlès, C. Weisman, Fast acoustic streaming in standing waves: Generation of an additional outer streaming cell, J. Acoust. Soc. Am. 134 (3) (2013) 1791-1801.

[30] C. Olivier, G. Penelet, G. Poignand, J. Gilbert, P. Lotton, Weakly nonlinear propagation in thermoacoustic engines: A numerical study of higher harmonics generation up to the appearance of shock waves, Acta Acust. United Acust. 101 (5) (2015) 941-949.

[31] B. Moudjed, V. Botton, D. Henry, H. Ben Hadid, J.-P. Garandet, Scaling and dimensional analysis of acoustic streaming jets, Phys. Fluids 26 (2014) 093602.

[32] M. Amari, V. Gusev, N. Joly, Temporal dynamics of the sound wind in acoustitron, Acta Acust. United Acust. 89 (2003) $1008-1024$.

[33] J.-C. Valière, S. Moreau, H. Bailliet, Development of laser techniques for acoustic boundary layer measurements. Part I: LDV signal processing for high acoustic displacements, Acta Acust. United Acust. 95 (2009) 585-594. 